\title{
$\ldots$
}

CDNF.8705163-2

\section{BROOKHAVEN NATIONÄL LABORATORY}

June 1987

BNL-39947

BNL- -39947

DE87 0].2868

\section{ELMUD: an ELectron MUon Detector for Higgs physics at the SSC}

\author{
Frank E. PAIGE \\ Physics Department \\ Brookhaven National Laboratory \\ Upton, NY 11973
}

\begin{abstract}
Requirements for detecting $H \rightarrow Z^{0} Z^{0} \rightarrow \ell^{+} \ell^{-} \ell^{+} \ell^{-}$at the SSC with $m_{H}=800 \mathrm{GeV}$ are explored. It is found a luminosity of $10^{34} \mathrm{~cm}^{-2} \mathrm{sec}^{-1}$ is needed but that the detector can have only a fast electromagnetic calorimeter plus a muon detector. Such a detector should be feasible at the required luminosity.
\end{abstract}

To appear in Proceedings of the Workshop "From Colliders to Supercolliders," Madison, WI, 11-22 May 1987.

This manuscript has been authored under contract number DE-AC02-76CH00016 with the U.S. Department of Energy. Accordingly, the U.S. Government retains a non-exclusive, royalty-free license to publish or reproduce the published form of this contribution, or allow others to do so, for U.S. Government purposes.r. 


\title{
ELMUD: an ELectron MUon Detector for Higgs physics at the SSC
}

\author{
Frank E. Paige \\ Physics Department \\ Brookhaven National Laboratory \\ Upton, NY $1: 1973$
}

\begin{abstract}
Requirements for detecting $H \rightarrow Z^{0} Z^{0} \rightarrow \ell^{+} \ell^{-} \ell^{+} \ell^{-}$at the SSC with $m_{H}=800 \mathrm{GeV}$ are explored. It is found a luminosity of $10^{34} \mathrm{~cm}^{-2} \mathrm{sec}^{-1}$ is needed but that the detector can have only a fast electromagnetic calorimeter plus a muon detector. Such a detector should be feasible at the required luminosity.
\end{abstract}

One of the major motivations for the SSC is the exploration of the origin of electroweak symmetry breaking. In the standard model this is due to one doublet of complex scalar Higgs fields; after symmetry breaking there remains a single physical particle $H$. If its mass is large enough, then its dominant decay modes are $W^{+} W^{-}$and $Z^{0} Z^{0}$. Three signatures have been extensively discussed:

$$
\begin{aligned}
& H \rightarrow Z^{0} Z^{0} \rightarrow \ell^{+} \ell^{-} \ell^{+} \ell^{-} \\
& H \rightarrow Z^{0} Z^{0} \rightarrow \ell^{+} \ell^{-} \nu \bar{\nu} \\
& H \rightarrow W^{+} W^{-} \rightarrow \bar{\ell}^{ \pm} \nu q \bar{q}
\end{aligned}
$$

where $\ell$ denotes either $e$ or $\mu$. Of these channels the decay into four charged leptons is certainly the cleanest, but because the branching ratio is so small, it becomes unobservable ${ }^{1}$ for masses above about $600 \mathrm{GeV}$ at $L=10^{33} \mathrm{~cm}^{-2} \mathrm{sec}^{-1}$. The decay into charged leptons and neutrinos ${ }^{2}$ has six times the branching ratio, but detection of it makes very stingent demands on the missing $p_{T}$ resolution. The third mode suffers from very large QCD backgrounds ${ }^{3}$, and while it may be possible to overcome these ${ }^{4}$, it is not easy to do so. It is therefore interesting to study detection of the charged lepton mode at higher luminosity.

The width of the Higgs grows like $G_{F} m_{H}^{3}$, reaching $250 \mathrm{GeV}$ at a mass of $800 \mathrm{GeV}$. Above about this mass perturbation theory ceases to be reliable, and longitudinally polarized $W^{ \pm}$and $Z^{0}$ become strongly interacting. The cross section ${ }^{5}$ for an $800 \mathrm{GeV}$ Higgs decaying into $\ell^{+} \ell^{-} \ell^{+} \ell^{-}$is $B \sigma=2.1 \times 10^{-39} \mathrm{~cm}^{2}$. Since there is a substantial background from the $q+\bar{q} \rightarrow Z^{0}+Z^{0}$ continuum, this is unobservable at $L=10^{33} \mathrm{~cm}^{-2} \mathrm{sec}^{-1}$ but would be observable at higher luminosity.

The SSC can probably be designed ${ }^{6}$ to produce $L=10^{34} \mathrm{~cm}^{-2} \mathrm{sec}^{-1}$. The question is what, if any, detectors can work at such high luminosity. Muon detection presumably remains feasible, since the muon absorber removes most of the rate, although triggering may be difficult and has not been addressed. Electromagnetic calormetry is the next easiest. Like all calorimetry it is insensitive to soft particles, and because electromagnetic showers are small, small towers can be used, minimizing pileup. Furthermore, 
there exist suitable fast materials such as $\mathrm{BaF}_{2}$, which emits part of its light with a time constant of $0.6 \mathrm{~ns}^{7}$. Hadronic calorimetry is more difficult; hadronic showers are much larger, and the known methods are slower. Measurement of missing $P T$ and tracking are both very difficult, the former because it is sensitive to global pileup and it requires small angle detectors, the latter because it is sensitive to soft particles.

For the $H$, or more generally for $Z^{0} Z^{0}$ pairs, it is not sufficient to detect only muons, since the factor of ten gain in rate is largely offset by the factor of four loss in branching ratio. Including also the $Z^{0} Z^{0} \rightarrow e^{+} e^{-} \mu^{+} \mu^{-}$channel gets $3 / 4$ of the possible rate. Assuming that the muons are identified and measured perfectly, the background comes from $Z^{0} j j$ events in which the jets fake electrons. This is similar to the $W^{ \pm} j j$ background for the Higgs; compared to it the signal is reduced by $B\left(Z^{0} \rightarrow e^{+} e^{-}\right)=.03$, while the background is reduced by the square of the probability of misidentifying a jet as an electron. Thus only modest electron identification is required. This leads one to consider ELMUD, a detector with a muon detector and a fast electromagnetic calorimeter but no hadronic calorimeter, tracking, or other electron identification. It appears that even such a limited detector can separate the $H \rightarrow \mu^{+} \mu^{-} e^{+} e^{-}$signal from the QCD background. The $e^{+} e^{-} e^{+} e^{-}$channel also has backgrounds from QCD jets. While these do not obviously overwhelm the signal, they are more difficult to analyse, and they are not considered here.

It is inlikely that the minimal version of ELMUD described here will ever be built at the SSC. One would want to add at least some hadronic calorimetry and perhaps also some tracking, if only to test its performance at lower luminosity. But a detector designed to run at $L=10^{34} \mathrm{~cm}^{-2} \mathrm{sec}^{-1}$ with muon detection, electromagnetic calorimetry, and hadronic calorimetry optimized for speed rather than precision or hermiticity appears to be an attractive possibility.

Some time ago ISAJET ${ }^{8}$ was used to generate a large sample of QCD jets, and it was found that the prompt muons from heavy quarks in these jets gave a negligible background to $Z^{0} \rightarrow \mu^{+} \mu^{-}$for $p_{T, Z}>50 \mathrm{GeV}$ even if no muon isolation criteria were imposed. This implies that the dominant background to the signals considered here comes from high- $p_{T} Z^{0}$ production accompanied by jets. To analyse this background, a total of 80000 events

$$
\begin{aligned}
& q+\bar{q} \rightarrow Z^{0}+g \\
& g+q \rightarrow Z^{0}+g
\end{aligned}
$$

with $Z^{0} \rightarrow \mu^{+}+\mu^{-}$have been generated using ISAJET 5.33 in two ranges, $200 \mathrm{GeV}<$ $p_{T, Z}<300 \mathrm{GeV}$ and $300 \mathrm{GeV}<p_{T, Z}<600 \mathrm{GeV}$ The initial and final state QCD radiation in these events produces multiple jets with approximately the correct rates. To reduce the amount of data, the events have been put into an idealized calorimeter with with $\Delta y=.05$ and $\Delta \phi=2.5^{\circ}$, and only those events have been kept in which the two highest $E_{T}$ towers make a $Z^{0}$ mass within $\pm 20 \mathrm{GeV}$.

In the analysis it is assumed that the muons are identified and measured perfectly. The electromagnetic calorimeter is treated in a very simple approximation based on CALSIM (which is distributed with ISAJET). It is taken to have uniform towers with $\Delta y=.05$ and $\Delta \phi=2.5^{\circ}$, and spreading of the showers is neglected, a not unreasonable approximation for towers of this size. The energy resolution is taken to be $\Delta E / E=$ $.15 / \sqrt{E}$ with $E$ measured in GeV. Charged hadrons are assumed to deposit no energy in the electromagnetic calorimeter, which is not correct. Adding the contribution from 
charged hadrons would increase the calculated probability of making a fake $Z^{0}$. But since the $\pi^{ \pm}$rate is only twice the $\pi^{0}$ rate, the probability of misidentifying a $\pi^{ \pm}$ as an electromagnetic shower need only be small compared to $50 \%$. In addition, the charged hadron contribution would make the isolation cut more effective than has been calculated. Clearly, however, a more realistic study of the detector is needed.

This idealized calorimeter is used to analyse three samples of events: the $H \rightarrow Z^{0} Z^{0}$ signal, the $Z^{0} Z^{0}$ continuum with $p_{T, Z}>200 \mathrm{GeV}$, and the $Z^{0} j j$ background. For each sample, the two highest $E_{T}$ towers in the electromagnetic calorimeter are selected and used as the $Z^{0} \rightarrow e^{+} e^{-}$candidate. The distribution for the mass of these are shown ir Fig. 1. Evidently the resolution introduced in the analysis produces some distortion of the $\pm 20 \mathrm{GeV}$ cut made in the generation of the $Z^{0} j j$ sample, but the effect is not large. The $Z^{0} Z^{0}$ mass distributions for the three samples are shown in Fig. 2; remember that the $Z^{0} j j$ background already contains cuts.

A series of cuts - which have not been optimized - are made to reduce the $Z^{0} j j$ background. The first cut, that mass of the second $Z^{0}$ be $94 \pm 8 \mathrm{GeV}$, gives Fig. 3 . The width of this cut was based on the width of the $Z^{0}$ in the signal. It seems likely that this width is dominated by the angular segmentation of the calorimeter and that better angular resolution would significantly improve the mass resolution. This has not been explored because it requires a correct treatment of shower spreading.

The second cut, $p_{T, Z}>200 \mathrm{GeV}$ for each $Z^{0}$, produces the mass distribution shown in Fig. 4. This cut reduces the $Z^{0} j j$ background, which contains many high-mass pairs formed from low- $p_{T}$ jets. It is also essential for reducing the $Z^{0} Z^{0}$ continuum, since the latter has a $t$-channel pole; it is included in generated continuum sample. No explicit cut is made on rapidity, but this cut implies approximately $\left|y_{Z}\right|<\mathbf{1 . 5}$.

The cut $p_{T, Z Z}<200 \mathrm{GeV}$ simply reflects the fact that a fake $Z^{0}$ generally carries less than the total $p_{T}$ of the jet and so does not balance the $p_{T}$ of the other $Z^{0}$. In addition, a cut is made on each of the towers forming the $Z^{0}$, requiring it to have $E_{T}>20 \mathrm{GeV}$. After these cuts, whose effects are shown in Fig. 5 , only $2 Z^{0} j j$ events survive out of 80000 generated.

Finally, a cut is made on the isolation of the electrons from $Z^{0} \rightarrow e^{+} e^{-}$; towers from jets should be less isolated. Since the electromagnetic towers are small, the pileup of soft events will contribute a substantial amount of $E_{T}$ to a region but very little to each individual tower. Therefore, this isolation cut is made by requiring that the highest additional tower within $\Delta R<.5$ of the electron candidates have $E_{T}<2 \mathrm{GeV}$. After this cut, shown in Fig. 6 , no $Z^{0} j j$ background events survive. The equivalent luminosity for the $300 \mathrm{GeV}<p_{T, z}<600 \mathrm{GeV}$ sample, corresponding to the range which dominates in the signal, is $1.3 \times 10^{40} \mathrm{~cm}^{-2}$, giving a $90 \%$ confidence upper limit for the $Z^{0} j j$ background of

$$
2.3(10 / 1.3)=17.7 \text { events } / \text { year }
$$

From Fig. 6 the Higgs signal is 33.2 events, while the $Z^{0} Z^{0}$ continuum in the mass range between 700 and $1000 \mathrm{GeV}$ is 33.4 events. This corresponds to 5.7 standard deviations, or 7.0 standard deviations if the $\mu^{+} \mu^{-} \mu^{+} \mu^{-}$channel is added with the same cuts.

Pileup may decrease the efficiency for the signal somewhat, primarily because of the isolation cut. Presumably the detector can be fast enough to reduce the pileup to the 20 or so events in a single bunch crossing. For an SSC minimum bias event there are about three $\pi^{0}$ per unit rapidity, each typically having $p_{T}=.5 \mathrm{GeV}$, giving for 20 events a total $E_{T}=1.2 \mathrm{GeV}$ within $\Delta R<.5$; this is spread over about 300 towers. Minijet events may be more of a problem, but the probability of a $\pi^{0}$ with $p_{T}>2 \mathrm{GeV}$ hitting 
the isolation cones, which cover only $2.5 \%$ of the phase space with $|y|<5$, should be small enough.

Pileup may also contribute to the background. The rate for two $Z^{0} \rightarrow \ell^{+} \ell^{-}$events with $p_{T}>200 \mathrm{GeV}$ occurring in the same bunch crossing is completely negligible, about .001 per year. For the $\mu^{+} \mu^{-} e^{+} e^{-}$channel superposition of jet events will increase the background. This needs to be calculated but is probably small, since the $Z^{0} \rightarrow \mu^{+} \mu^{-}$ event already contains QCD radiation with a scale set by $p_{T, z}$. For the $e^{+} e^{-} e^{+} e^{-}$ channel background can arise from the superposition of several relatively soft jets; the rate for this has not been estimated.

It should be evident that the results obtained here depend on the tails of distributions whose validity is not now known. However, the requirement on the probability of misidentifying a jet as an electron is not very stringent, and the background is almost eliminated without the last isolation cut. Additional cuts might be made to reduce both the $Z^{0} j j$ and the $Z^{0} Z^{0}$ continuum backgrounds. For example, the $Z^{0}$ from $B$ have primarily longitudinal polarization and $a \sin ^{2} \theta$ decay distribution, while the $Z^{0}$ from the $Z^{0} j j$ and the $Z^{0} Z^{0}$ continum have primarily transverse polarization and a $1+\cos ^{2} \theta$ distribution. Thus it seems promising that ELMUD could find the desired signal. What other physics it could do remains to be studied.

This work was supported in part by the United States Department of Energy under Contract DE-AC02-76CH00016.

\section{REFERENCES}

1. E. Wang, these proceedings.

2. R. Cahn and M. Chanowitz, Phys. Rev. Lett. $\underline{56}, 1327$ (1986).

3. J.F. Gunion, Z. Kunzst, and M. Scldate, Phys. Lett. 163B, 389 (1985);

S.D. Ellis, R. K!eiss, and W.J. Stirling, Phys. Lett. 163B, 261 (1985).

4. S.D. Protopopescu, BNL-38620 (1986), to appear in Snowmass 86.

5. E. Eichten, I Hinchliffe, K. Lane, and C. Quigg, Rev. Mod. Phys. $\underline{56}, 579$ (1984).

6. M. Tigner, private communication.

7. C.L. Woody and D.F. Anderson, Fermilab-Pub-87/42.

8. F.E. Paige and S.D. Protopopescu, BNL-38774 (1986), , to appear in Snowmass 86. 


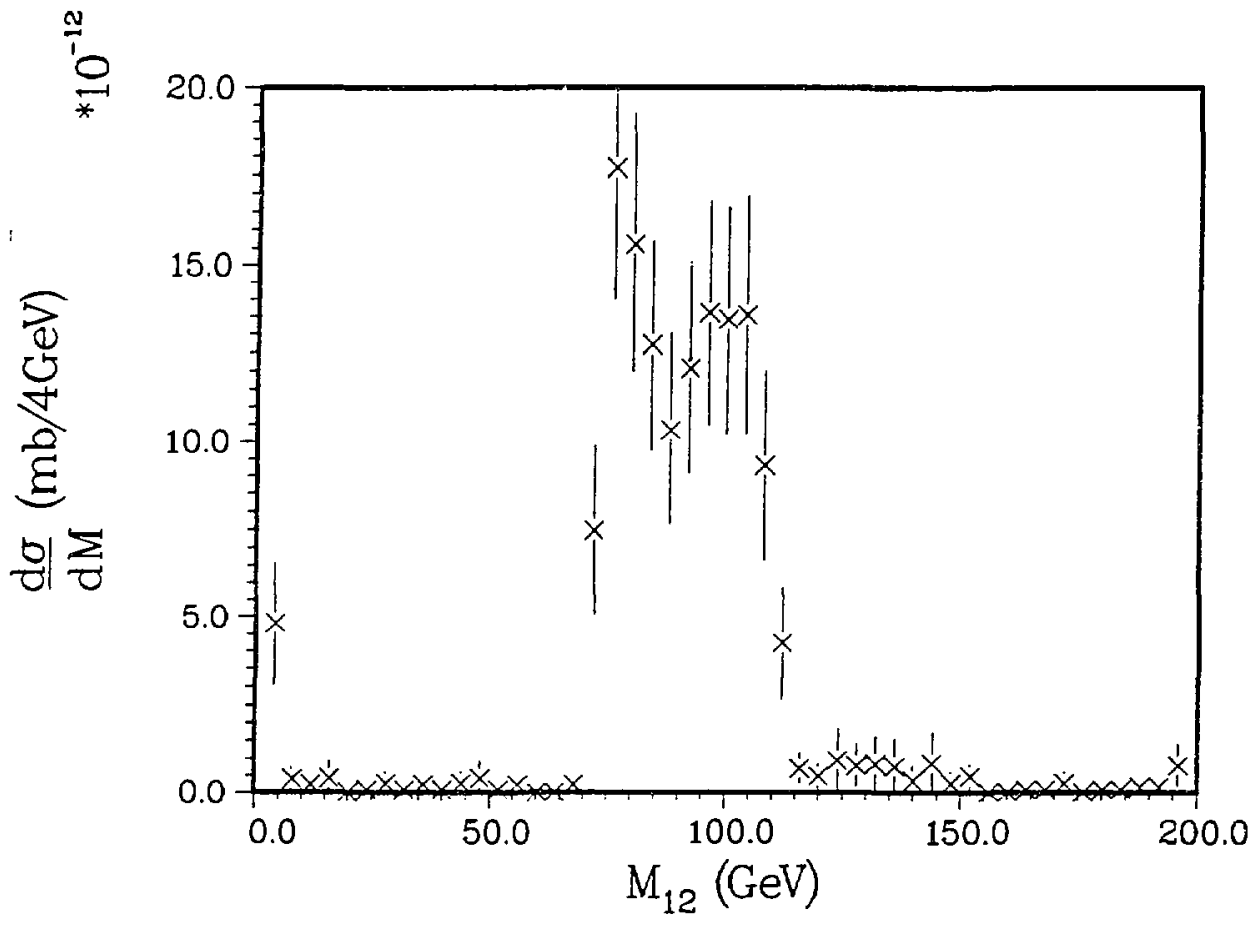

Fig. 1: Distribution of masses from two highest towers in the $Z^{0} j j$ sample after inclusion of detector resolution.

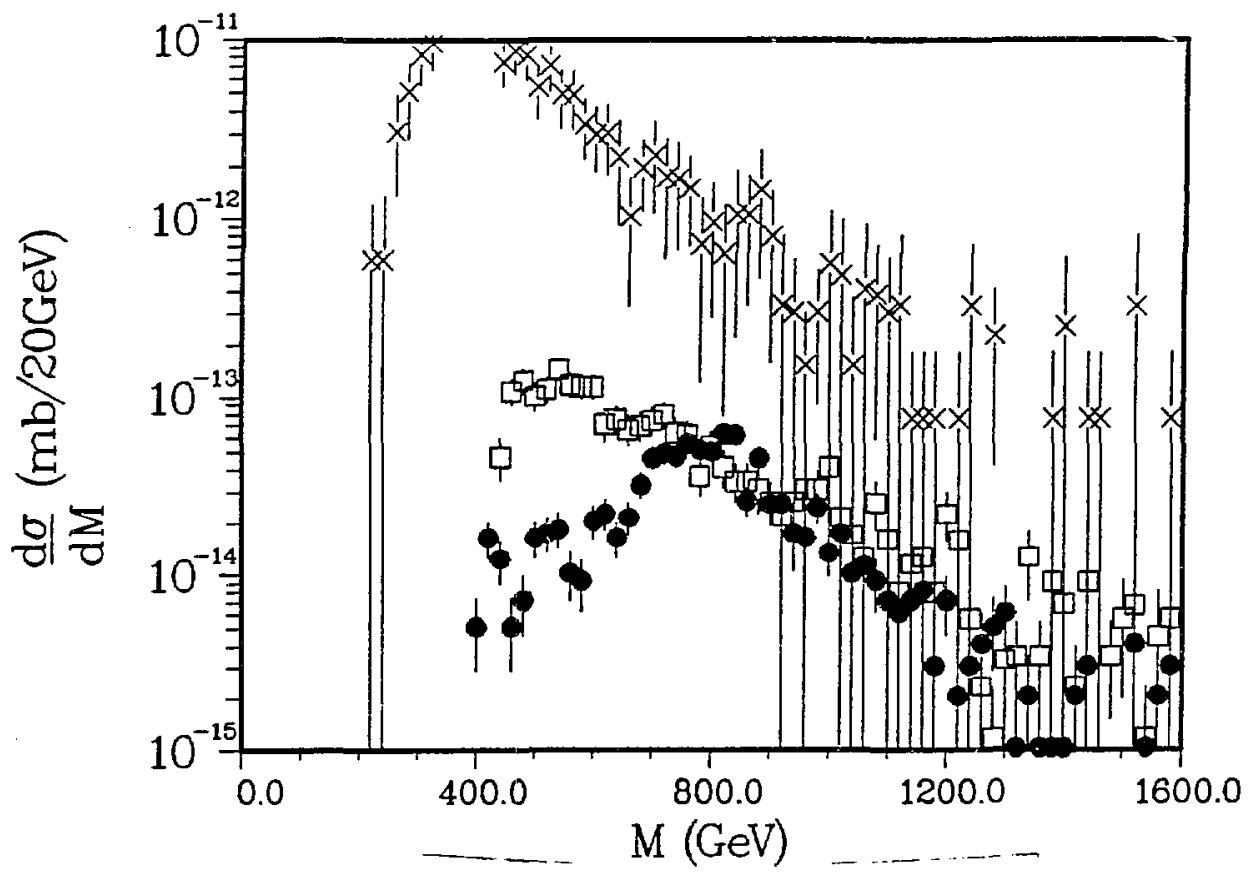

Fig 2: Distribution of $Z^{0} Z^{0}$ masses before any cuts. Circles: $H \rightarrow Z^{0} Z^{0}$. Squares: $Z^{0} Z^{0}$ continuum. Crosses: $Z^{0} j j$ background. 


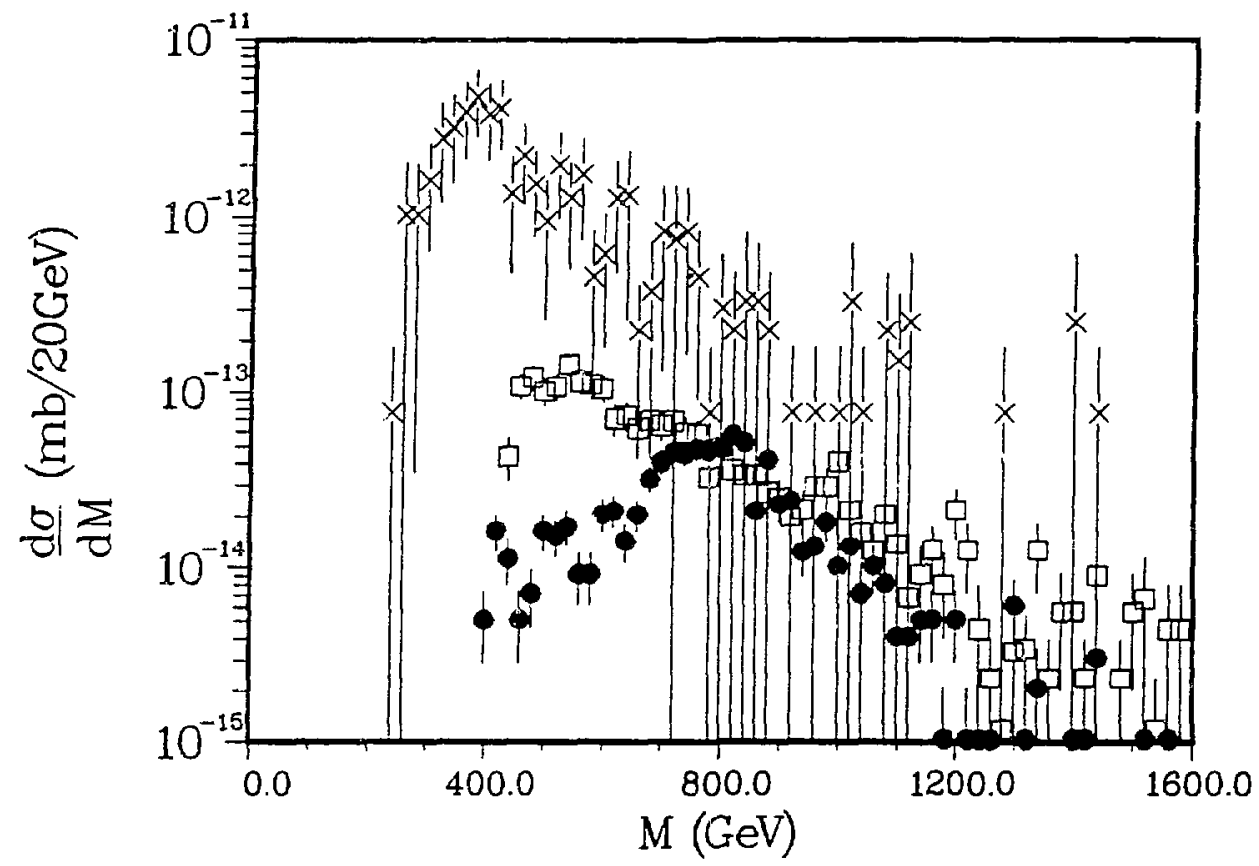

Fig 3: Distribution of $Z^{0} Z^{0}$ masses after $\pm 8 \mathrm{GeV}$ mass cut.

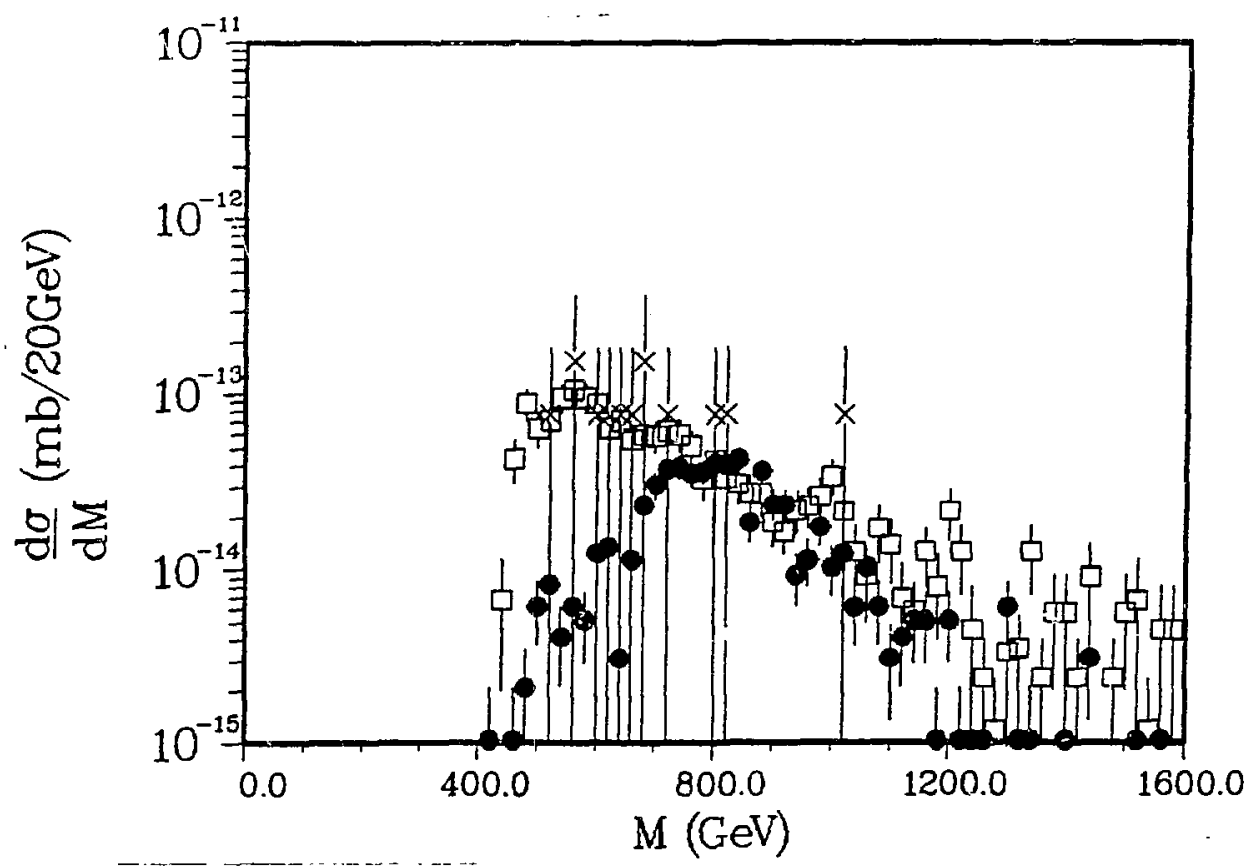

Fig 4: Distribution of $Z^{0} Z^{0}$ masses after $p_{T, Z}>200 \mathrm{GeV}$ cut. 


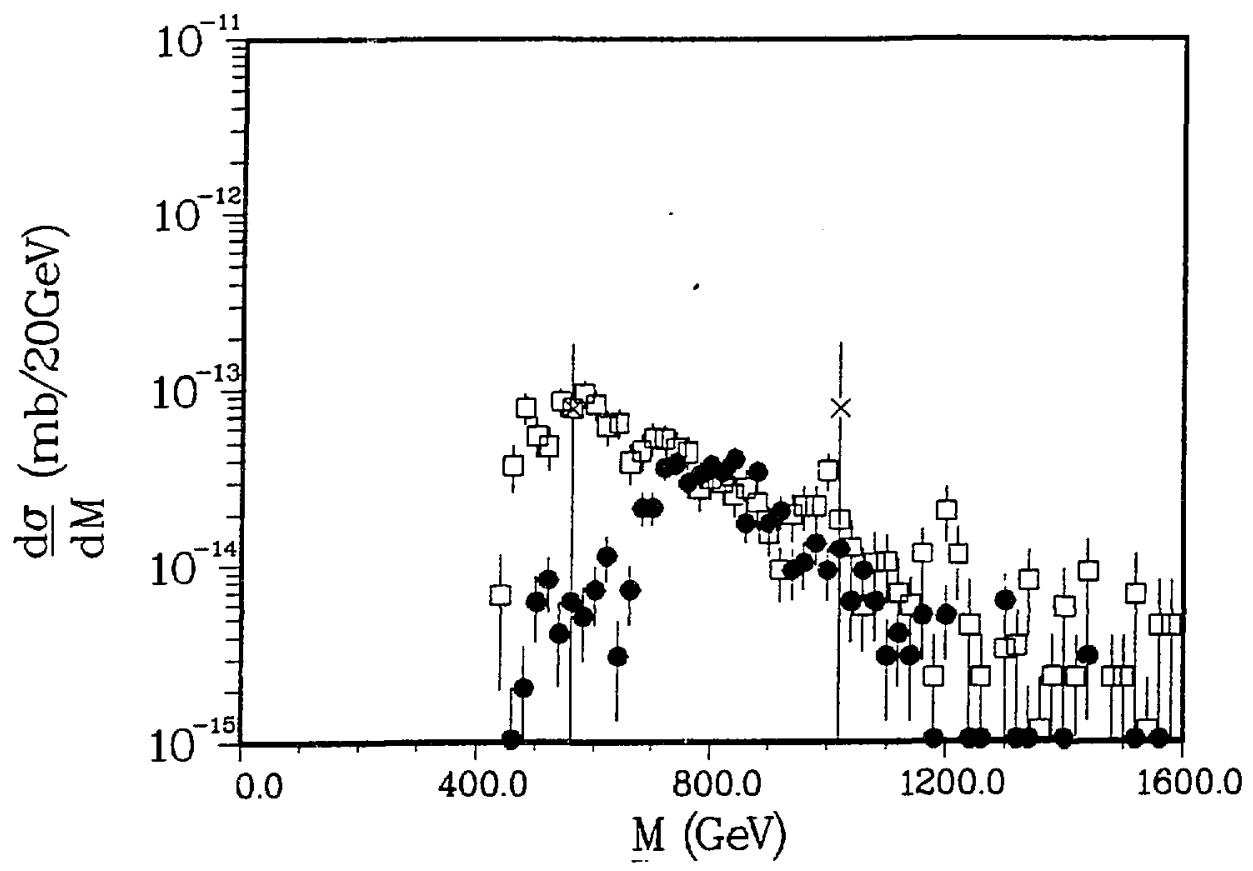

Fig 5: Distribution of $Z^{0} Z^{0}$ masses after $p_{T, Z Z}<200 \mathrm{GeV}$ and $E_{T}>20 \mathrm{GeV}$ cuts.

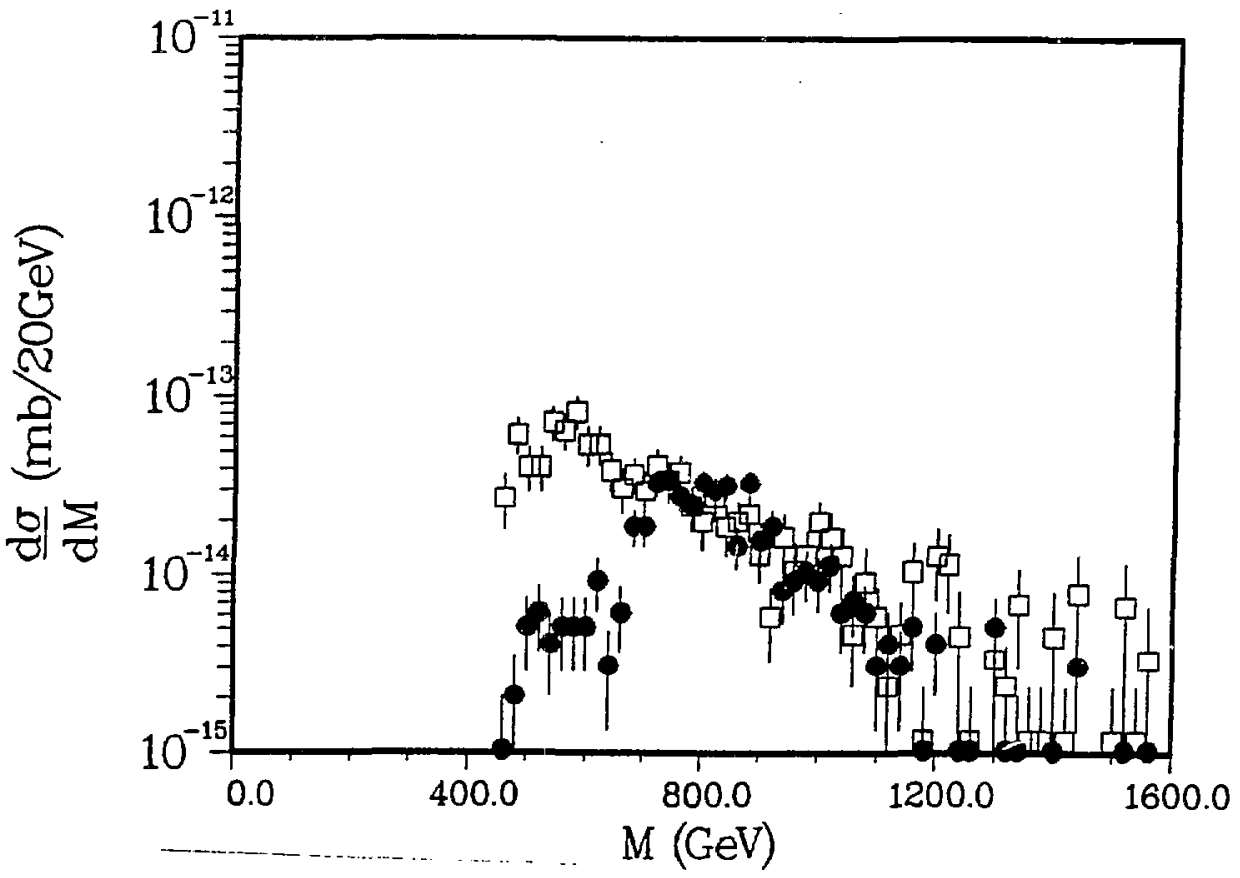

Fig 6: Distribution of $Z^{0} Z^{0}$ masses after isolation cut. 